Relations industrielles

Industrial Relations

\title{
L'horaire variable de travail, par Jean-François Baudraz, Montréal, Les Éditions d'Organisation, Bordas-Dunod, 1971, 135 \\ pp.
}

\section{Laurent Bélanger}

\section{Volume 29, numéro 1, 1974}

URI : https://id.erudit.org/iderudit/028490ar

DOI : https://doi.org/10.7202/028490ar

Aller au sommaire du numéro

Éditeur(s)

Département des relations industrielles de l'Université Laval

ISSN

0034-379X (imprimé)

1703-8138 (numérique)

Découvrir la revue

Citer ce compte rendu

Bélanger, L. (1974). Compte rendu de [L'horaire variable de travail, par Jean-François Baudraz, Montréal, Les Éditions d'Organisation, Bordas-Dunod, 1971, 135 pp.] Relations industrielles / Industrial Relations, 29(1), 227-228. https://doi.org/10.7202/028490ar

Tous droits réservés (c) Département des relations industrielles de l'Université Laval, 1974
Ce document est protégé par la loi sur le droit d'auteur. L’utilisation des services d'Érudit (y compris la reproduction) est assujettie à sa politique d'utilisation que vous pouvez consulter en ligne.

https://apropos.erudit.org/fr/usagers/politique-dutilisation/ 
of that period could deal equally well with new and as yet not quite clearly perceived problems within the same institutional framework and with the same methods. 》

It is becoming increasingly evident that the upward shift in decision making that is involved in organization to organization bargaining can result in serious alienation of the workers and an erosion of the union's influence on the individual. Union-management relations at the plant level are frequently non existent under such a system. While Joint Works Councils, whether imposed by statute or voluntarily undertaken, may help to fill a vacuum in labourmanagement relations at the local level, Malles indicates that they have had little effect on the decision-making process at the level of the enterprise. Thus although they may appear as the epitome of industrial democracy when viewed at a distance, in practice they seem to have added nothing to the gains achieved by collective bargaining on the North American pattern.

Chapter IV also deals briefly with the question of worker participation on boards of directors, with various attempts at capital formation in workers' hands, and with recent experiments in the organization of work, notably those replacing assembly line operations with small production groups. These experiments are an interesting response to the problem of worker morale and are based on the theory that job satisfaction will bring significant productivity gains. In the final section, the author considers the impact of the multi-national corporation on industrial relations and the particular challenge this presents to the national and international labour movement. Could any topic be more relevant to current Canadian concerns?

\section{Shirley B. GOLDENBERG}

\section{McGill University}

L'horaire variable de travail, par JeanFrançois Baudraz, Montréal, Les Editions d'organisation, Bordas-Dunod, $1971,135 \mathrm{pp}$.

L'horaire flexible de travail constitue une pratique récente en matière d'amé- nagement du temps de travail. Il se distingue des horaires conventionnels (fixes ou décalés ou spéciaux) en ce sens qu'il confie à l'employé la responsabilité d'utiliser sagement son temps à l'intérieur de sa journée de travail en dehors d'une période de temps bloqué. Au même moment où cette pratique s'installe progressivement dans les administrations et les entreprises, elle fait l'objet de nombreux articles et volumes décrivant ses aspects techniques et psycho-sociologiques. L'étude de Jean-François Baudraz s'inscrit dans ce courant. L'auteur nous fait part de ses observations et de ses réflexions sur le sujet, puisqu'à titre de conseiller il a eu l'occasion d'aider à l'introduction d'un horaire variable dans deux entreprises, celle de la Banque Cantonale Vaudoise à Lausanne et celle de Ciba-Geigy à Monthey. Pour bien situer ces deux expériences, l'auteur retrace l'idée de 1'horaire variable, établit des distinctions entre ce type d'horaire et les horaires conventionnels. Il décrit ensuite les problèmes d'organisation du travail, du contrôle du temps-travail et de l'information des cadres et du personnel. Cette description constitue l'aspect technique de son étude. La deuxime partie qui se veut plutôt analytique porte sur une dimension qui intéresse la direction des entreprises et plus particulièrement les préposés à la gestion du personnel : c'est l'étude des avantages et des inconvénients de cette nouvelle pratique pour les individus et pour les entreprises. Pour l'individu, l'horaire variable facilite les déplacements en élargissant le choix des moyens de transport, diminue la tension nerveuse due à la peur d'arriver en retard, à la peur de ne pas disposer d'assez de temps pour s'occuper de ses affaires personnelles. Cette pratique accorde à l'individu une plus grande liberté d'action. Pour l'entreprise, c'est un moyen efficace de réduire les absences, les retards et les heures supplémentaires, d'améliorer le climat social. Par ailleurs, elle force les cadres à remettre en cause la conception qu'ils se font de l'autorité et de son exercice puisque l'horaire variable exige une plus grande délégation de pouvoir.

Cette pratique comporte aussi des inconvénients. On ne peut l'étendre pour le moment à toutes les entreprises ou à toutes les divisions d'une entreprise. Le calcul des heures de travail, s'il se fait 
par lecteur optique relié à un ordinaentreprises. Par contre, l'informatique teur, implique des dépenses que ne peuvent encourir les petites et les moyennes n'est pas le seul moyen pour établir un décompte des heures et la paie des employés. Il existe d'autres instruments de calcul qui rendent possible l'extension de cette pratique à la petite et moyenne entreprise.

L'implantation de l'horaire flexible à l'échelle de la société industrielle créerait une occasion de revalorisation de l'individu au travail par un accroissement de responsabilités et par un élargissement des choix personnels, et cela, à un moment où les exigences de la technologie continuent à influencer les comportements en milieu de travail. C'est une réflexion globale qui se dégage de l'ensemble de l'ouvrage et c'est ce qui, à mon sens, en constitue l'aspect le plus intéressant.

Laurent BELANGER

Université Laval

Workers' Control, A Reader on Labor and Social Change, Edited by Gerry Hunnius, G. David Gardon and John Case, New York, Random House, 1973, 493 pp.

Le sujet qu'abordent les auteurs, «Le contrôle ouvrier», est loin d'être un sujet nouveau bien qu'il n'ait jamais été tellement à la mode au Canada et aux Etats-Unis.

En effet, le problème de la démocratisation des rapports du travail au moyen d'une gestion de l'entreprise par les travailleurs a toujours été au centre des théories socialistes et c'est une demande qui s'inscrit dans la tradition du mouvement ouvrier international.

L'intérêt que nous portons à ce livre est d'autant plus grand que peu de travaux portant sur le contrôle ouvrier ont été publiés ou sont disponibles en Amérique du Nord. De plus, les auteurs ont su éviter le danger d'offrir une option qui pourrait nous apparaître de peu d'intérêt eu égard à la valeur de notre système de relations du travail.
C'est pourquoi, ce livre, très bien conçu, comprend d'abord une critique sévère de la négociation collective dont "le rôle aujourd'hui est de fournir un cadre institutionnel rigide à la lutte des classes ». On fait ressortir les limites de ce système institutionnel. On porte également un jugement sévère sur le syndicalisme américain «qui sert les intérêts des grandes firmes » et qui contribue à intégrer la force ouvrière au système. Nous retrouvons sur cette question d'excellents articles de Stanley Aronowitz et de Daniel Bell.

Après une critique du système de négociation collective nord-américain, les auteurs présentent certains modèles contemporains de participation et d'autogestion. Ainsi les idées de « contrôle ouvrier 》 perdent ce qu'elles pouvaient avoir d'obscures pour s'offrir à nous comme une réalité. La perspective critique est toujours présente aussi bien dans l'analyse du système yougoslave que suédois que dans celle du modèle de cogestion allemand, lequel est perçu par Helmut Schauer comme une « rationalisation des relations sociales existantes » et non comme une «suppression du pouvoir capitaliste $\gg$.

Et dans une dernière partie les auteurs présentent le «contrôle ouvrier» comme une des stratégies de changement. Le «contrôle ouvrier» ne saurait être perçu comme une fin en soi, ni comme une simple réforme industrielle. Il ne trouve sa vraie signification que s'il est placé dans «la perspective stratégique d'une révolution politique et sociale ». Les articles de André Gorz et de Ernest Mandel sont des plus intéressants sur cette question.

Il est nécessaire avant tout de retenir que le «contrôle ouvrier» se situe totalement en dehors des théories participationnistes actuelles et tel que présenté dans ce livre il est une revendication anticapitaliste. On voit rnal comment il pourrait être absorbé ou digéré par le système au même titre que des augmentations de salaires ou des avantages sociaux accrus. C'est tout le problème de la relation entre stratégie et fin qui est posé. 